\title{
Distribution characteristics of Staphylococcus spp. in different phases of periprosthetic joint infection: A review (Review)
}

\author{
GEYONG GUO $^{1}$, JIAXING WANG ${ }^{1}$, YANAN YOU ${ }^{2}$, JIAQI TAN ${ }^{1}$ and HAO SHEN ${ }^{1}$ \\ ${ }^{1}$ Department of Orthopedic Surgery, Shanghai Jiao Tong University Affiliated Sixth People's Hospital; \\ ${ }^{2}$ Department of Obstetrics, Fudan University Affiliated Obstetrics and Gynecology Hospital, Shanghai 200233, P.R. China
}

Received December 22, 2015; Accepted January 26, 2017

DOI: $10.3892 /$ etm.2017.4300

\begin{abstract}
Periprosthetic joint infection (PJI) is a devastating condition and Staphylococcus spp. are the predominant pathogens responsible, particularly coagulase-negative staphylococci (CoNS) and Staphylococcus aureus. The aim of the present systematic review was to evaluate the distribution characteristics of specific Staphylococcus spp. in different PJI phases, reveal the effect of pathogens' feature on their distribution and suggest recommendations for antibiotic treatment of Staphylococcal PJI. The present systematic review was performed using PubMed and EMBASE databases with the aim to identify existing literature that presented the spectrum of Staphylococcus spp. that occur in PJI. Once inclusion and exclusion criteria were applied, 20 cohort studies involving 3,344 cases in 3,199 patients were included. The predominant pathogen involved in PJI was indicated to be CoNS (31.2\%), followed by $S$. aureus (28.8\%). This trend was more apparent in hip replacement procedures. In addition, almost equal proportions of CoNS and S. aureus (28.6 and $30.0 \%$, respectively) were indicated in the delayed phase. CoNS (36.6\%) were the predominant identified organism in the early phase, whereas S. aureus $(38.3 \%)$ occurred primarily in the late phase. In PJI caused by $S$. aureus, the number of cases of methicillin-sensitive Staphylococcus aureus (MSSA) was 2.5 -fold greater than that of methicillin-resistant Staphylococcus aureus (MRSA). MRSA occurred predominantly in the early phase, whereas MSSA was largely observed in the delayed and late phases. With regards to antibiotic treatment, the feature of various pathogens and the phases of PJI were the primary considerations. The present review provides useful information for clinical practice and scientific research of PJI.
\end{abstract}

Correspondence to: Dr Hao Shen, Department of Orthopedic Surgery, Shanghai Jiao Tong University Affiliated Sixth People's Hospital, 600 Yishan Road, Shanghai 200233, P.R. China E-mail: shenhao7212@sina.com

Key words: periprosthetic joint infection, coagulase-negative staphylococci, Staphylococcus aureus, phases, antibiotic treatment

\author{
Contents \\ 1. Introduction \\ 2. Materials and methods \\ 3. Results \\ 4. Summary and interpretation of main results \\ 5. Agreement and disagreement with other studies \\ 6. Antibiotic treatment \\ 7. Limitations \\ 8. Conclusions
}

\section{Introduction}

Various studies have indicated that the incidence of periprosthetic joint infection (PJI), following total joint arthroplasty (TJA), ranged between 0.5 and $2 \%$ for primary knee arthroplasty and 0.2 to $1.3 \%$ for primary hip replacement (1-5); however, when considering revision surgery, the infection risks were elevated markedly $(4,6)$. For the past few decades, various effective strategies, such as the use of laminar airflow, body exhaust suits, prophylactic antibiotics (intravenous antibiotics and antibiotic-loaded cement) and minimizing operating room traffic, have all contributed to reducing infection rates in general $(4,6-10)$. Despite this, the total number of infections following TJA have been significantly increasing over the past few years $(1,3,11)$ as a result of the continuously increasing arthroplasties required among the elderly $(10,12,13)$. Once established, PJI is a disastrous condition for patients and is a serious economic burden to the healthcare system $(1,3,4,6,7,10,14-16)$.

As previously reported, Staphylococcus spp. are the predominant causative organisms associated with PJI, particularly coagulase-negative staphylococci (CoNS) and Staphylococcus aureus, which comprise $50-80 \%$ of all isolates (12,16-25). However, the variant species of Staphylococcus differ from one another in virulence, antibiotic resistance and biofilm formation ability, thus have individual distribution characteristics in different phases of PJI. On the basis of the PJI classification system proposed by Zimmerli et al (26), the early phase PJI, presenting within 3 months, are typically caused by virulent bacteria such as $S$. aureus and gram-negative bacilli, whereas delayed PJI, presenting between 3 and 24 months, are commonly caused by less virulent pathogens such as CoNS and Propionibacterium acnes (26). 
As a critical pathogen of CoNS, the incidence of methicillin-resistant Staphylococcus epidermidis (MRSE) $(2,9,23,27-29)$ has increased significantly in PJI over the past two decades (27,29-33), which may be associated with the use of prophylactic antibiotics (32). However, whether MRSE is able to cause the distribution changes in the phases of PJI has not been studied until now. Similarly, S. aureus may be classified into two categories: Methicillin-susceptible S. aureus (MSSA) and methicillin-resistant S. aureus (MRSA). Due to differences in antibiotic resistance, discrepancies appear in the occurrence, treatment and prognosis of MSSA and MRSA (19,34-36). However, few studies have reported the discrepancy of the incidence between MSSA and MRSA in PJI $(15,34,35)$. In the present review, literature concerning PJI in recent decades was reviewed to interpret the distribution characteristics of Staphylococcus spp. in different PJI phases and investigate the influence of pathogens' virulence and antibiotic resistance on distribution changes. Furthermore, the present review suggests recommendations for antibiotic treatment, dependent on the feature of various pathogens and the phases of PJI.

\section{Materials and methods}

Search methods. The present systematic review of published studies was performed without language limitation using the most common databases, PubMed (https://www.ncbi. nlm.nih.gov/pubmed/) and EMBASE (https:/www.embase. com/\#search). The key words used were: 'Staphylococcus', 'infection', 'revision', 'knee arthroplasty/replacement', 'hip arthroplasty/replacement', 'joint arthroplasty/replacement', 'arthroplasty, replacement, knee/hip' and 'arthroplasty, replacement'. Studies published prior to June 30, 2015 were searched.

Inclusion and exclusion criteria. Inclusion criteria were: Patients undergoing a PJI or deep infection following knee or hip arthroplasty; and microbiology of the infected arthroplasty that involved staphylococcal species. Exclusion criteria were: Animal or in vitro experiments and the surgical sites of PJI or deep infection did not include the knee or hip; the spectrum of staphylococci was not reported; and data extraction from the studies was difficult.

Data collection, extraction, management and analysis. The titles and abstracts of all studies were screened carefully and the potentially eligible studies were elected by one author. Subsequently, two reviewers critically analyzed the full texts of the selected studies. Every contradiction was resolved by group discussion directed by one author.

In the present review, the classification system for PJI proposed by Zimmerli et al (26) was used. According to this classification system, PJI should be classified into three phases: early, presenting within 3 months; delayed, between 3 and 24 months; and late, over 24 months $(1,2,11,13,25$, $26,31,37)$. Relevant data of included studies were extracted independently by two investigators and verified by another. The extracted data included: Authors, year of publication, study design, study period, number of cases and patients, mean age, gender, surgery site, phase of PJI and spectrum of causative microorganisms.
All data were collected in specifically designed Microsoft Office Excel spreadsheets. According to the same inclusion criteria proposed by Zimmerli et al (26), data was pooled from various included studies in order to compensate for the deficiency of the single study.

In order to observe the distribution characteristics of Staphylococcus spp. in the knee and hip, stratification analysis was performed according to the surgery site type (hip or knee). Subsequently, when the distribution characteristics of bacteria in different infection phases were analyzed, the previous inclusion criteria were modified by adding the following criterions: i) PJI was classified according to Zimmerli et al (26) or could be converted to the criteria; and ii) having corresponding pathogens in three different phases of PJI. Consequently, eligible documents were selected among the included studies. Analogously, when the percentages of MSSA and MRSA were compared, studies were chosen based on the availability of the classification information of $S$. aureus.

Statistical methods. SAS software (Release 8.02 TS Level 02M0; SAS Institute, Inc., Cary, NC, USA) was used to perform statistical analysis. The frequencies and percentages of the pathogens were calculated and analyzed in the included studies and merged data. The Pearson $\chi^{2}$ test was used to assess the distribution of microbiological findings. $\mathrm{P}<0.05$ was considered to represent significant differences.

\section{Results}

Description of the selected studies. Due to duplications between PubMed and EMBASE, 447 articles were excluded among 1,025 eligible studies identified in the initial literature retrieval. Subsequently, 520 were refused by screening the titles and abstracts. The remaining 58 studies, including an additional 19 studies added from a reference review, were in entirety read and 56 articles were eliminated for not meeting the inclusion criteria. Subsequently, the remaining 20 articles were included in the present systematic review after one unsuitable research article was rejected as the necessary data extraction was difficult. According to the new inclusion criteria, four studies were selected and used to observe the distribution characteristics of Staphylococcus spp. in different PJI phases. A total of 10 studies were used to analyze the categorization features of S. aureus in PJI. A flow chart of the selection process is shown in Fig. 1.

General characteristics of included studies. The 20 included studies involved 3,344 cases in 3,199 patients following joint arthroplasty, including 1,630 knee, 1,700 hip and 14 others, such as ankle, shoulder and elbow cases. All of these identified studies were retrospective/prospective cohort studies in this search. The sample size of the included studies varied from 15 to 785 cases between the years of 1974 to 2013. The general characteristics and patient demographics of the included studies are displayed in Table I.

Among the 20 studies, six studies (1,8,33,38-40) reported knee cases, five $(9,32,41-43)$ reported hip cases and nine $(2,6,12,13,19,20,27,28,44)$ reported knee and hip cases. Of the nine studies, four articles $(6,12,28,44)$ were divided into two categories of knee and hip cases on the basis of the type 


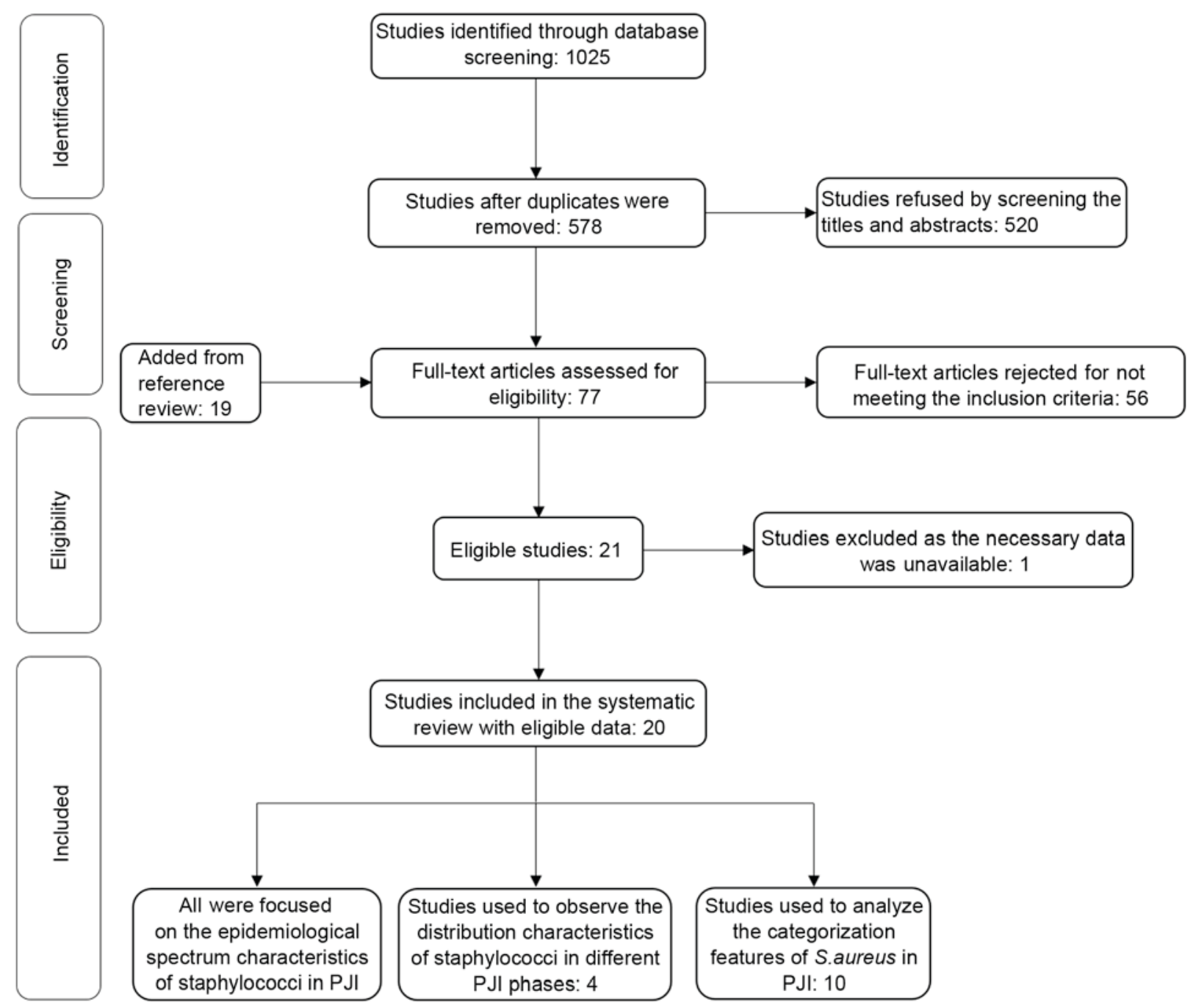

Figure 1. Flow chart of selection process in the systematic review. PJI, periprosthetic joint infection.

of staphylococci, whereas the other five studies $(2,13,19,20,27)$ were not. Furthermore, a small fraction of polymicrobial infection that occurred in a number of studies $(1,2,9,12,13,20$, $27,38,39,41)$ was converted to the monomicrobial infection for calculating the percentage of sole pathogens in the present review.

Incidences of Staphylococcus in 20 included studies. In the 20 included studies, CoNS (11.1-67.1\%) and $S$. aureus (0.0-54.9\%) were the predominant organisms in PJI following joint arthroplasty, as presented in Table II. The pooled rates of CoNS and S. aureus were 31.2 and $28.8 \%$, respectively, which together accounted for $60.0 \%$ of the total isolated organisms. Furthermore, the rates of CoNS isolated from knee cases ranged from 7.1 to $66.7 \%$ in 10 studies, with a pooled rate of $28.1 \%$. However, the incidences of S. aureus varied from 0.0 to $50.0 \%$. The merged rate was $27.5 \%$. Likewise, CoNS were cultured from hip cases in nine studies, with rates ranging from 13.6 to $67.1 \%$. The pooled rate was $34.7 \%$. S. aureus incidence in PJI ranged from 12.9 to $51.7 \%$, with a merged rate of $25.4 \%$ in the hip. It was apparent that the most frequently encountered microorganism was CoNS, followed by $S$. aureus in total knee arthroplasty (TKA) or total hip arthroplasty (THA). Overall, the distributions of CoNS and S. aureus were similar (31.2 and $28.8 \%$, respectively) in PJI. However, CoNS was indicated to be more common in the hip (34.7\%) than in the knee $(28.1 \%$; $\mathrm{P}=0.004)$.

Incidences of Staphylococcus in different phases of PJI. As classified by the occurring time of PJI in Table III, 524 infected cases in four studies $(1,2,28,39)$ were identified and met the new inclusion criteria. The occurrence rates of infections were indicated to be $29.2 \%$ in the early phase, $41.4 \%$ in the delayed phase, and $29.4 \%$ in the late phase. Of the three phases, CoNS and $S$. aureus (41.6 and $41.7 \%$, respectively) were distributed predominantly in the delayed phase. In terms of the sole microorganism detected within each respective phase there was a similar proportion of CoNS and S. aureus (28.6 and 30.0\%, respectively) in the delayed phase. When comparing CoNS and S. aureus, CoNS (36.6\%) was determined as the predominant organism in the early phase, whereas S. aureus $(38.3 \%)$ was detected primarily in the late phase $(\mathrm{P}<0.001)$.

Comparison of MSSA and MRSA in 10 included studies. Among the included studies, 10 studies $(2,13,19,27,28,33,40,41$, $43,44)$, involving 510 cases in 1,587 patients infected with 
Table I. General characteristics and patient demographics in 20 included studies.

\begin{tabular}{|c|c|c|c|c|c|c|c|c|}
\hline Author, year & $\begin{array}{l}\text { Study } \\
\text { period }\end{array}$ & $\begin{array}{l}\text { Study } \\
\text { design }\end{array}$ & $\begin{array}{c}\text { Cases } \\
(\mathrm{N})\end{array}$ & $\begin{array}{l}\text { Patients } \\
(\mathrm{N})\end{array}$ & $\begin{array}{c}\text { Gender } \\
(\mathrm{F} / \mathrm{M})\end{array}$ & $\begin{array}{l}\text { Mean } \\
\text { age } \\
\text { (years) }\end{array}$ & $\begin{array}{c}\text { Surgery site } \\
(\mathrm{N}) \\
\begin{array}{c}\text { Knee/Hip/ } \\
\text { Others }\end{array}\end{array}$ & (Refs.) \\
\hline Wilde et al, 1988 & $1980-1987$ & PCS & 15 & 15 & $7 / 8$ & 68.0 & $15 / 0 / 0$ & (39) \\
\hline Burger et al, 1991 & 1976-1988 & RCS & 39 & 39 & ND & ND & $39 / 0 / 0$ & (38) \\
\hline Giulieri et al, 2004 & $1985-2001$ & PCS & 63 & 60 & $20 / 40$ & 72.0 & $0 / 63 / 0$ & $(42)$ \\
\hline Ip et al, 2005 & $1995-2003$ & RCS & 36 & 36 & $12 / 24$ & 70.0 & $14 / 22 / 0$ & (28) \\
\hline Fulkerson et al, 2006 & $1991-2003$ & RCS & $194^{\mathrm{a}}$ & 146 & $82 / 64$ & 66.0 & $84 / 110 / 0$ & (12) \\
\hline Phillips et al, 2006 & $1987-2001$ & PCS & 75 & 75 & $38 / 37$ & 67.6 & $41 / 34 / 0$ & (2) \\
\hline Rafiq et al, 2006 & $1974-2005$ & RCS & 255 & 255 & ND & ND & $0 / 255 / 0$ & (9) \\
\hline Moran et al, 2007 & $1998-2003$ & RCS & 112 & 112 & $50 / 62$ & 68.8 & $51 / 52 / 9$ & $(27)$ \\
\hline Sharma et al, 2008 & 1999-2006 & RCS & $248^{\mathrm{a}}$ & 147 & ND & ND & $108 / 140 / 0$ & (6) \\
\hline Stefansdottir et al, 2009 & $1986-2000$ & $\mathrm{RCS}$ & 426 & 426 & $244 / 182$ & $\begin{array}{l}\text { Primary } 68.1 \\
\text { revision } 70.9\end{array}$ & $426 / 0 / 0$ & (1) \\
\hline Teterycz et al, 2010 & 1996-2008 & RCS & 82 & 82 & $39 / 43$ & 69.0 & $29 / 52 / 1$ & (19) \\
\hline Nickinson et al, 2010 & 1994-2008 & RCS & 121 & 121 & $60 / 61$ & 71.0 & $121 / 0 / 0$ & (8) \\
\hline Tsai et al, 2015 & $2006-2011$ & $\mathrm{RCS}$ & 144 & 144 & $81 / 63$ & 68.9 & $88 / 52 / 4$ & (13) \\
\hline Kuiper et al, 2013 & 2004-2009 & RCS & 91 & 91 & $54 / 37$ & 70.0 & $29 / 62 / 0$ & (20) \\
\hline Lutro et al, 2014 & 1993-2007 & PCS & 278 & 278 & $188 / 90$ & 67.6 & $0 / 278 / 0$ & (32) \\
\hline Ibrahim et al, 2014 & $2000-2008$ & PCS & 125 & 125 & $74 / 51$ & 68.0 & $0 / 125 / 0$ & (41) \\
\hline Bjerke-Kroll et al, 2014 & 1998-2011 & RCS & 785 & 793 & $355 / 438$ & $\begin{array}{l}\text { TKA } 65.6 \\
\text { THA } 65.3\end{array}$ & $390 / 395 / 0$ & (44) \\
\hline Holmberg et al, 2015 & $2000-2008$ & PCS & 145 & 144 & $62 / 82$ & 70.0 & $145 / 0 / 0$ & (40) \\
\hline Matsumoto et al, 2015 & $2000-2010$ & RCS & 50 & 50 & $12 / 38$ & 71.1 & $50 / 0 / 0$ & (33) \\
\hline Triantafyllopoulos et al, 2015 & $2001-2013$ & $\mathrm{RCS}$ & 60 & 60 & $31 / 29$ & 64.9 & $0 / 60 / 0$ & (43) \\
\hline Total & & & 3,344 & 3,199 & & & $1,630 / 1,700 / 14$ & \\
\hline
\end{tabular}

${ }^{a}$ Cases were positive specimens isolated from knee or hip. F, female; M, male; PCS, prospective cohort study; RCS, retrospective cohort study; ND, no data; primary, primary arthroplasty; revision, revision arthroplasty; TKA, total knee arthroplasty; THA, total hip arthroplasty.

S. aureus, were selected to provide the classification information of $S$. aureus. The ratios of MSSA and MRSA varied from 9 to $633 \%$. The merged ratio was $262 \%$. A clear trend was observed where the number of MSSA was 2.5-fold greater than that of MRSA in PJI caused by S. aureus. Fig. 2 summarizes the detailed information.

\section{Summary and interpretation of main results}

CoNS may be more common than S. aureus, particularly in hip PJI. As a principal part of the skin and mucous membrane microflora $(8,45,46)$, constituting $65-90 \%$ of all staphylococci, CoNS are commonly encountered in clinical settings, particularly during orthopedic surgery. In an article researching intraoperative bacterial contamination during joint replacement, Davis et al (45) discovered CoNS were involved in $76 \%$ of 776 gathered samples. Equally, CoNS were identified in $77 \%$ of contaminated femoral heads, which were donated during primary hip arthroplasty (47).

Arciola et al (21) and Montanaro et al (22) conducted two large studies concerning orthopaedic infections. In infections associated with medical devices, the prevalence of
Staphylococcus epidermidis was observed to have increased from 31.5\% in the previous collection (between 2000 and 2003) to $39.0 \%$ in the new collection (between 2007 and 2011), and the incidence of $S$. epidermidis was greater than $S$. aureus (39.0 and $31.7 \%$, respectively) (16,22). These studies revealed that CoNS were progressively prevailing when compared with S. aureus (22). Moreover, S. epidermidis was the dominating bacteria identified in infections associated with knee and hip arthroplasties, whereas $S$. aureus was predominantly exhibited in infections associated with no medical devices and fracture fixation devices (22). These evidences suggest that CoNS were more common than $S$. aureus in PJI.

Compared with primary or revision TKA, patients undergoing primary or revision THA tended to be older, sicker and required a longer length of stay in hospital $(36,48)$, indicating that patients who underwent TKA generally had a stronger ability to resist CoNS, a less virulent pathogen, when compared with patients who underwent THA. Moreover, in THA infection, Lutro et al (32) demonstrated that CoNS (41\%) were more frequent than S. aureus (19\%) and exhibited a significant increase in resistance to nearly all antibiotics (except for linezolid and vancomycin), whereas $S$. aureus and other bacteria exhibited 
Table II. Incidence of Staphylococcus spp. in 20 included studies.

\begin{tabular}{|c|c|c|c|c|c|}
\hline \multirow[b]{2}{*}{ Author, year } & \multirow[b]{2}{*}{ Cases (N) } & \multicolumn{3}{|c|}{ Causative organism, N (\%) } & \multirow[b]{2}{*}{ (Refs.) } \\
\hline & & CoNS & S. aureus & Others $^{\mathrm{a}}$ & \\
\hline \multicolumn{6}{|l|}{ Knee and hip } \\
\hline Phillips et al, 2006 & 75 & $27(36.0)$ & $22(29.3)$ & $26(34.7)$ & (2) \\
\hline Moran et al, 2007 & 112 & $47(42.0)$ & $53(47.3)$ & $12(10.7)$ & (27) \\
\hline Teterycz et al, 2010 & 82 & $39(47.6)$ & $43(52.4)$ & $0(0.0)$ & (19) \\
\hline Tsai et al, 2015 & 144 & $24(16.7)$ & 43 (29.9) & $77(53.5)$ & (13) \\
\hline Kuiper et al, 2013 & 91 & $13(14.3)$ & $50(54.9)$ & $28(30.8)$ & (20) \\
\hline \multicolumn{6}{|l|}{ Knee } \\
\hline Wilde et al, 1988 & 15 & $10(66.7)$ & $0(0.0)$ & $5(33.3)$ & (39) \\
\hline Burger et al, 1991 & 39 & $13(33.3)$ & $19(48.7)$ & $7(17.9)$ & (38) \\
\hline Ip et al, 2005 & 14 & $1(7.1)$ & $6(42.9)$ & $7(50.0)$ & (28) \\
\hline Fulkerson et al, 2006 & $84^{\mathrm{b}}$ & $30(35.7)$ & $27(32.1)$ & $27(32.1)$ & (12) \\
\hline Sharma et al, 2008 & $108^{\mathrm{b}}$ & $28(25.9)$ & $26(24.1)$ & $54(50.0)$ & (6) \\
\hline Stefansdottir et al, 2009 & 426 & $131(30.8)$ & $134(31.5)$ & $161(37.8)$ & (1) \\
\hline Nickinson et al, 2010 & 121 & $59(48.8)$ & $16(13.2)$ & $46(38.0)$ & (8) \\
\hline Bjerke-Kroll et al, 2014 & 390 & $76(19.5)$ & 77 (19.7) & $237(60.8)$ & (44) \\
\hline Holmberg et al, 2015 & 145 & $33(22.8)$ & $53(36.6)$ & $59(40.7)$ & $(40)$ \\
\hline Matsumoto et al, 2015 & 50 & $10(20.0)$ & $25(50.0)$ & $15(30.0)$ & (33) \\
\hline Total & 1,392 & $391(28.1)$ & $383(27.5)$ & $618(44.4)$ & \\
\hline \multicolumn{6}{|l|}{ Hip } \\
\hline Giulieri et al, 2004 & 63 & $11(17.5)$ & $27(42.9)$ & $25(39.7)$ & (42) \\
\hline Ip et al, 2005 & 22 & $3(13.6)$ & $6(27.3)$ & $13(59.1)$ & (28) \\
\hline Fulkerson et al, 2006 & $110^{\mathrm{b}}$ & $31(28.2)$ & $40(36.4)$ & $39(35.5)$ & (12) \\
\hline Rafiq et al, 2006 & 255 & $171(67.1)$ & $33(12.9)$ & $51(20.0)$ & (9) \\
\hline Sharma et al, 2008 & $140^{\mathrm{b}}$ & $50(35.7)$ & $27(19.3)$ & $63(45.0)$ & (6) \\
\hline Lutro et al, 2014 & 278 & $113(40.6)$ & $53(19.1)$ & $112(40.3)$ & $(32)$ \\
\hline Ibrahim et al, 2014 & 125 & $35(28.0)$ & $49(39.2)$ & $41(32.8)$ & (41) \\
\hline Bjerke-Kroll et al, 2014 & 395 & $77(19.5)$ & $102(25.8)$ & $216(54.7)$ & (44) \\
\hline Triantafyllopoulos et al, 2015 & 60 & $11(18.3)$ & $31(51.7)$ & $18(30.0)$ & (43) \\
\hline Total & 1,448 & $502(34.7)$ & $368(25.4)$ & $578(39.9)$ & \\
\hline Total & 3,344 & $1,043(31.2)$ & $962(28.8)$ & $1,339(40.0)$ & \\
\hline
\end{tabular}

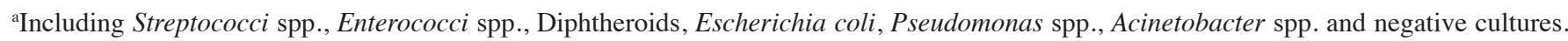
${ }^{\mathrm{b}}$ Cases were positive specimens isolated from knee or hip. CoNS, coagulase-negative staphylococci; S. aureus, Staphylococcus aureus.

no significant changes in susceptibility patterns from the years 1993 to 2007, in Norway. Although there were some regional differences (32), the changes in susceptibility patterns of CoNS may have a contribution to the result that CoNS were more commonly observed in the hip than the knee in the present review.

CoNS were the prevalent pathogen in early phase PJI, whereas $S$. aureus occurred predominantly in the late phase. Previous reports have demonstrated that contamination in the operating room and haematogenous dissemination through sepsis or transient bacteremia were the two primary routes of infection following joint replacement $(17,18,45)$. Early and delayed PJI were likely to be caused by intraoperative contamination, whereas the most common source of late infection was haematogenous spread $(25,26,49)$.
In order to reduce intraoperative contamination risk, antibiotic prophylaxis has been routinely applied in clinical practice in addition to applying physical measures (7). However, among 131 Staphylococcus-positive specimens, Sharma et al (6) indicated that 6 of the 53 S.aureus isolates were methicillin-resistant and all MSSA isolates were cephalothin- and cefazolin-sensitive, whereas $65 \%$ of the CoNS isolates were resistant to cephalothin. Similarly, Nickinson et al (8) concluded that almost all antibiotics were more effective against $S$. aureus when compared with CoNS.

For another, Teterycz et al (19) reported that bacteremia was detected in MRSA and MSSA in 163 episodes and not in CoNS infections. Other researchers have identified a similar phenomenon (11). All these studies indicated that $S$. aureus were more virulent and exhibited a shorter incubation 
time (19), greater ability to form biofilms and were able to adapt locally (50) when compared with CoNS $(23,28)$.

Consequently, $S$. aureus relatively rarely caused infections in the early phase following surgery due to the comprehensive perioperative protocol (51). However, with the subsiding of precaution in the late phase, for example the decrease of antibiotic concentration from antibiotic-loaded cement $(52,53)$, S. aureus may rapidly cause infections.

Additionally, biofilm formation has been demonstrated as a mechanism in establishing antibiotic resistance $(16,28,54,55)$. $S$. aureus were frequently associated with acute clinical presentation produced by numerous intra-articular planktonic pathogens $(25,54,55)$ and were indicated to be the most frequent microbe that produces a biofilm (54), suggesting that $S$. aureus may be detected and treated in the early phase. However, the biofilms developed by CoNS, particularly S. epidermidis, have often exhibited negative results on blood bacterial culture and have only been identified after the removal and culture of the implant (23). Therefore, CoNS may have invaded the implant and caused PJI in the early phase, prior to any noticeable symptoms of PJI. Taking this into account, we hypothesized that these factors may explain why CoNS were more common in the early phase of PJI and S.aureus were more common in the late phase of PJI.

MSSA comprised up to $70 \%$ of all S. aureus in PJI. According to the present findings and the reports of other studies $(15,32,34,56)$, the isolated rate of MSSA in PJI is much higher than that of MRSA. However, MRSA has been indicated to be more resistant to antibiotics (57) and has a worse patient prognosis than MSSA (19,34,35). Lora-Tamayo et al (34) demonstrated that $88 \%$ of MRSA-PJI treatment failure occurred during the early stage of treatment; whereas 50\% of MSSA-PJI treatment failure occurred following cessation of therapy. Alternatively, Liu et al (57) showed that MSSA harbored more diverse and abundant virulence factor genes than MRSA. Due to offsetting the costs associated with virulence and methicillin resistance $(57,58)$, the toxin expression may decrease in MRSA (57). This implied that MSSA was more virulent than MRSA. Furthermore, the hematogenous PJI was indicated to be caused by MSSA in $88 \%$ of episodes (34) and the late phase of PJI was thought to be the result of haematogenous spread $(25,26,49)$. This may explain why hematogenous PJI or late phase PJI always developed suddenly and progressed rapidly.

From previous implantation to arthroplasty infection onset, the median time delay of MRSA and MSSA was 16 and 361 days, respectively, as Teterycz et al (19) described. In a joint registry review, Siddiqui et al (59) also clearly indicated that $44 \%$ of early infections following primary TKA were the result of MRSA. Therefore, when combined with the findings of the present review, S. aureus occurred predominantly in the delayed and late phases of PJI, and MSSA was in the majority. Therefore, we had reason to conclude that MRSA may be the predominant bacteria retrieved in the early phase, and MSSA in the delayed and late phases of PJI caused by S. aureus.

\section{Agreement and disagreement with other studies}

Consistent with previous studies $(2,6,8,9,17,23,25,29,39,60)$, the most frequently encountered isolates in the present review 


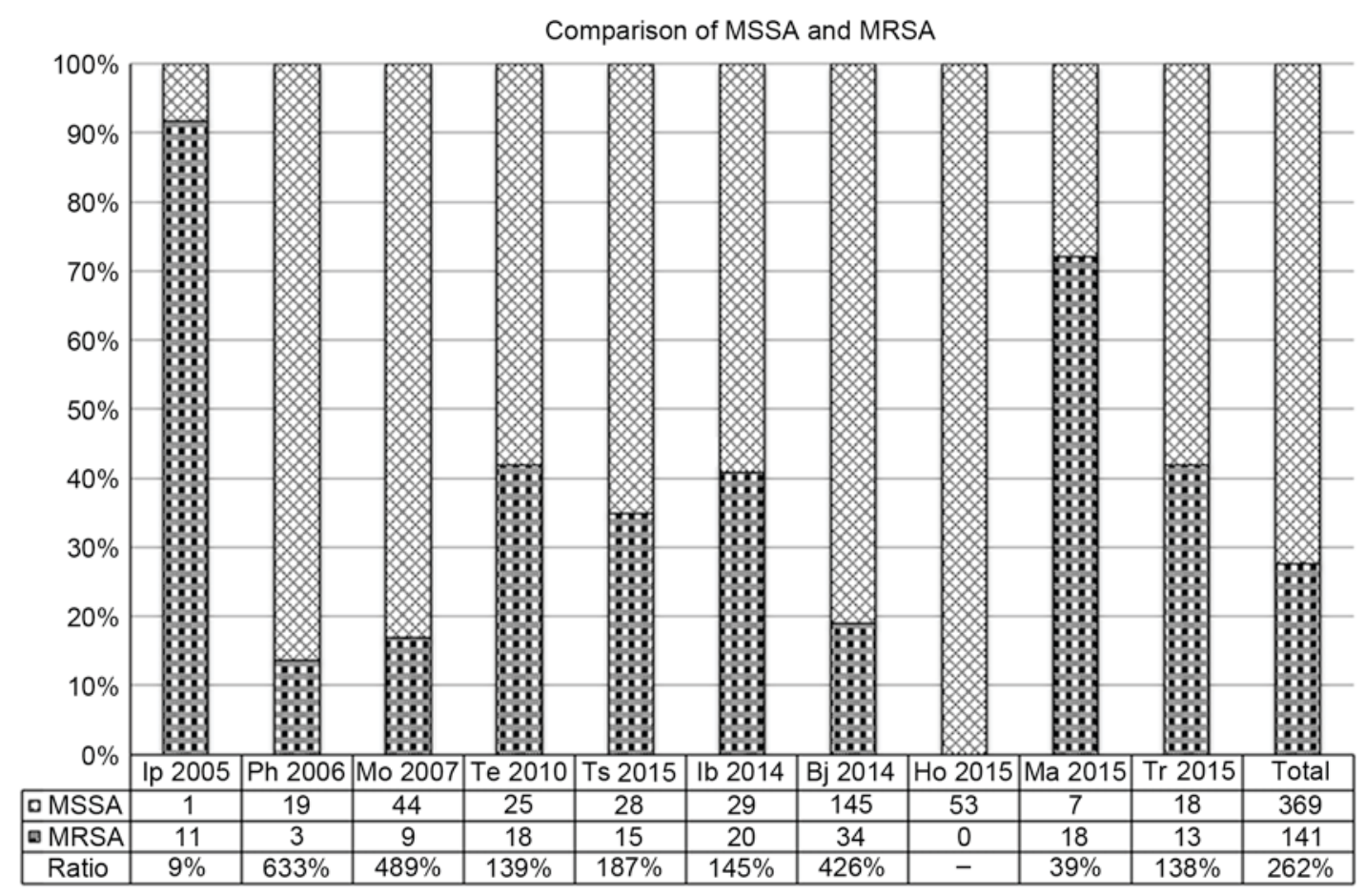

Figure 2. Comparison of MSSA and MRSA in 10 included studies. MSSA, methicillin-susceptible Staphylococcus aureus; MRSA, methicillin-resistant Staphylococcus aureus; Ip, Ip et al (28); Ph, Phillips et al (2); Mo, Moran et al (27); Te, Teterycz et al (19); Ts, Tsai et al (13); Ib, Ibrahim et al (41); Bj, Bjerke-Kroll et al (44); Ho, Holmber et al (40); Ma, Matsumoto et al (33); Tr, Triantafyllopoulos et al (43).

were CoNS, followed by $S$. aureus. Although multiple studies have been conducted $(1,12,27,28,31,41,61)$ revealing that PJI was predominantly caused by $S$. aureus when compared with CoNS, the overall proportions were similar in the majority of these studies. The slight disparity may be due to the differences of infection control policies in different regions or hospitals $(24,32,62)$.

Findings of the present review revealed the distribution characteristics of CoNS and S. aureus in hip and knee arthroplasties were in broad agreement with previously published data $(1,8,9,32,41,60)$. However, the present review did not further discuss the feature modifications of Staphylococcus spp. in PJI longitudinally due to lack of sufficient data. As previously reported, the incidences of CoNS and S. aureus reversed over time in hip and knee arthroplasties $(8,9,17)$. For example, Muilwijk et al (17) and Rafiq et al (9) demonstrated that $S$. aureus appeared to become more prevalent following hip arthroplasties, with CoNS decreasing in prevalence, which was reversed in the infected knee replacements (8). However, in 2014, Aggarwal et al (62) and Bjerke-Kroll et al (44) demonstrated that there were no significant differences in CoNS and S. aureus when comparing hip to knee arthroplasties. Only patients who underwent two-stage revision were included in the study conducted by Bjerke-Kroll et al (44), which may lead to a degree of bias in predicting the overall distribution characteristics of Staphylococcus spp. in hip and knee cases.

In different phases of PJI, the present review indicated that the delayed phase of infection (41.4\%) was the most common infection, followed by late phase $(29.4 \%)$ and early phase (29.2\%) infection. This result was associated with the PJI classification system. Phillips et al (2) revealed that $36 \%$ of patients exhibited late phase PJI and 35\% exhibited delayed phase PJI, when PJI occurred greater than one year after surgery were classified as late infections. However, of all the infections, $52 \%$ arose between 3 months and 2 years (delayed phase) and $19 \%$ after 2 years (late phase), when the classification system applied in our review was used (2).

Ascione et al (31) indicated that in 159 patients with early or delayed PJI, S. aureus (39\%) was the principal cause of early infections, followed by CoNS (13\%), using the same classification system for PJI that was chosen in the present review. This study disagreed with the findings of the present review. Under similar conditions, the results of the present review did not agree with Zimmerli et al (26), who proposed that the early phase PJI were typically caused by virulent bacteria, whereas delayed phase PJI were commonly caused by less virulent pathogens. As described earlier, subclinical syndrome always occurred at the beginning of the PJI caused by CoNS, which may make it difficult to diagnose during the early (27). Therefore, the correct diagnosis may be delayed if the clinical manifestations alone are depended upon, which may result in certain differences in various studies. This suggests that more rigorous methods are required for the early diagnosis of PJI $(27,63)$. Even though the classification system used in the present review is universally used $(2,25)$, specific disadvantages of this classification system are apparent. In particular, Sendi et al (11) stated that this classification system was not optimal for distinguishing exogenous and haematogenous PJI, when PJI was caused by $S$. aureus $<2$ years after surgery.

In a large retrospective and multicenter study of PJI caused by S. aureus, $77 \%$ of cases were caused by MSSA and $23 \%$ of episodes were caused by MRSA among a total of 345 episodes (34). In 2007, Salgado et al (15) also revealed that 
$24 \%$ of cases were the result of MSSA and $9 \%$ of cases were a result of MRSA in THA or TKA PJI. These were similar to the findings of the present review. But perhaps because MRSA typically results in a worse prognosis than MSSA and predominantly occurred in the early phase of PJI, various studies $(15,59,64)$ have investigated MRSA-PJI, whereas little is known regarding MSSA-PJI at present. Therefore, the present review indicated that MRSA occurred primarily in the early phase of PJI and MSSA occurred predominantly in the delayed and late phases of PJI, which may aid the understanding of the distribution characteristics of these two strains in PJI and aid the development of reasonable antibiotic treatment strategies.

\section{Antibiotic treatment}

Deficiencies in the current antibiotic-resistant bacteria treatment strategy. In 2010, Teterycz et al (19) demonstrated that MRSA had the worst outcome in arthroplasty infections, which was cured in $39 \%$ of episodes, compared with $60 \%$ of MSSA episodes and $77 \%$ of CoNS episodes. Therefore, multiple prevention and treatment measures have been primarily used to treat $S$. aureus (or MRSA) in clinical practice (56), rather than CoNS. However, in the present review, $31.2 \%$ of PJI cases were indicated to be caused by CoNS. Furthermore, MRSE isolated from PJI has become an increasingly popular pathogen over the past decades. For example, James et al (30) revealed that a quarter of patients had MRSE on their skin prior to hip arthroplasties and 18/30 cultures of $S$. epidermidis were isolated, of which 12 were positive for MRSE in 1994. Moran et al (27) indicated in their retrospective review of 112 patients that $60 \%$ of CoNS cases ( $47 \%$ of patients) were methicillin-resistant and $88 \%$ of CoNS cases were penicillin-resistant. The changes in the distribution characteristics of pathogens in PJI emphasized the requirement of choice for appropriate treatment protocols.

Choice of antibiotic treatment. Although multiple factors must be considered prior to deciding on an antibiotic treatment strategy, the most critical factors are the bacterial species and the phases of PJI.

The feature of pathogens has been suggested to be a strong indicator of the outcome of antibiotic treatment $(3,28,62)$. Joshy et al (65) indicated that PJI caused by MRSA or MRSE, following TKA, exhibited significantly lower satisfactory outcomes (18 and 67\%, respectively) when compared to those caused by MSSA. To prevent these refractory infections, we suggest that prophylactic antibiotics should include vancomycin and gentamicin, a theory supported by Sharma et al (6). In their clinical study, all Gram-positive bacteria, such as $S$. epidermidis and $S$. aureus (12), were vancomycin-sensitive and almost all Gram-negative organisms were gentamicin-sensitive in 248 isolates from revision hip and knee arthroplasties (6).

Unfortunately, various studies have revealed that although CoNS and MRSA infections decreased after patients received vancomycin prophylaxis (14), MSSA infections were more likely to be develop. Similarly, MRSA infections were more common if patients received cefazolin prophylaxis alone $(7,14)$. This phenomenon may be due to reduced antimicrobial spectrum and decreased efficacy of vancomycin as compared to cefazolin for CoNS and S. aureus (14). Therefore, for staphylococcal PJI cases, the combined application of antibiotics was recommended to improve the therapeutic effect (66), prevent the occurrence of antibiotic resistance and reduce systemic toxicity $(67,68)$.

With the emergence of vancomycin-resistant Enterococcus spp. (69), there was an urgent requirement to take a number of effective measures to slow the progression of antibiotic-resistant strains. Therefore, a number of medical researchers recommended that vancomycin should only be administered to patients possessing a known drug-resistant bacteria carrier status $(7,28)$ with the exception of the combined application of antibiotics. Additionally, various studies had demonstrated that early screening of MRSA $(56,64)$ and MRSE (46) may significantly reduce the incidence of these resistant strains in PJI, thus screening for MRSA and MRSE in patients prior to surgery was necessary to establish a suitable antibiotic prophylaxis and treatment policy.

The empiric antibiotic therapy should be guided by the phases of PJI until the correct culture results are available $(12,37)$. In the present review, MRSA and CoNS were the prevalent genus identified in the early and delayed phase of PJI and the infections were treated with vancomycin and surgical debridement, which have been recommended by other studies $(1,10,12)$. In late phase PJI, the majority of PJI cases were the result of hematogenous seeding by MSSA. For infections in this phase, Fulkerson et al (12) have suggested combination therapy with cefazolin and gentamicin, whereas Lora-Tamayo et al (34) recommended the combination of quinolones plus rifampin. However, regardless of the phase of PJI, resistant pathogens, such as MRSA and MRSE, typically exhibit a high rate of treatment failure (70), particularly in the delayed and late phase PJI. Hence, in certain studies, revision (one-stage or two-stage) coupled with systemic antibiotics was often necessary $(8,10,41,65)$. When confronted with incurable PJI, arthrodesis, resection arthroplasty and amputation (65) may have to be adopted for survival. The sensitivity of the causative organisms towards antibiotics was the basis of PJI treatment.

\section{Limitations}

The present review exhibited several limitations. The sample size used may be too small for the effective classification of PJI and S. aureus; therefore, the present results, in conjunction with conclusions from alternative studies, were analyzed carefully in order to attain a reliable conclusion. Furthermore, the present review analyzed exclusively staphylococcal infections due to CoNS and $S$. aureus and did not factor in alternative pathogens that were categorized as 'Others'. Hence, the present conclusion was unserviceable for PJI caused by other pathogens. Additionally, a small fraction of polymicrobial infection in some included studies was integrated into the monomicrobial PJI for analyzing the frequency of sole pathogens. Due to the limited information of mixed infections, we were unable to determine the characteristic of polymicrobial infection in PJI.

\section{Conclusions}

PJI is a serious clinical problem for patients and doctors. In the present systematic review, the pooled rates of CoNS and S. aureus accounted for $60.0 \%$ of the isolated organisms in 20 
included studies. CoNS were identified to be a more common cause of PJI than S. aureus, particularly in the cases of hip PJI. In different phases of PJI, CoNS were the prevalent genus in the early phase, whereas $S$. aureus was primarily observed in the late phase, although CoNS and S. aureus were exhibited in a similar proportion in the delayed phase. In PJI caused by S. aureus, MSSA occurred more frequently. Moreover, MRSA occurred predominantly in the early phase, whereas MSSA was more common in the delayed and late phases.

To prevent PJI, the combined application of antibiotics and comprehensive early carrier status screening of MRSA and MRSE are recommended. In terms of antibiotic treatment, vancomycin and gentamicin should be included in prophylactic antibiotics in order to cover Gram-positive and Gram-negative organisms. Vancomycin and surgical debridement were often used in the early and delayed phase of PJI and the combination of cefazolin and gentamicin or the combination of quinolones and rifampin were proposed in the late phase, prior to the final culture results. Although there are limitations in this review, our results may be useful for clinical practice and further scientific research of PJI.

\section{Acknowledgements}

This work was supported by a grant from the National Natural Science Foundation of China (grant no. 81472108).

\section{References}

1. Stefánsdóttir A, Johansson D, Knutson K, Lidgren L and Robertsson O: Microbiology of the infected knee arthroplasty: Report from the Swedish Knee Arthroplasty Register on 426 surgically revised cases. Scand J Infect Dis 41: 831-840, 2009.

2. Phillips JE, Crane TP, Noy M, Elliott TS and Grimer RJ: The incidence of deep prosthetic infections in a specialist orthopaedic hospital: A 15-year prospective survey. J Bone Joint Surg Br 88: 943-948, 2006.

3. Marculescu CE and Cantey JR: Polymicrobial prosthetic joint infections: Risk factors and outcome. Clin Orthop Relat Res 466: 1397-1404, 2008.

4. Lentino JR: Prosthetic joint infections: Bane of orthopedists, challenge for infectious disease specialists. Clin Infect Dis 36 : 1157-1161, 2003.

5. Rodriguez-Merchan EC: The infected knee prosthesis. Eur J Orthop Surg Traumatol 21: 467-478, 2011.

6. Sharma D, Douglas J, Coulter C, Weinrauch P and Crawford R: Microbiology of infected arthroplasty: Implications for empiric peri-operative antibiotics. J Orthop Surg (Hong Kong) 16: 339-342, 2008.

7. Sewick A, Makani A, Wu C, O'Donnell J, Baldwin KD and Lee GC: Does dual antibiotic prophylaxis better prevent surgical site infections in total joint arthroplasty? Clin Orthop Relat Res 470: 2702-2707, 2012.

8. Nickinson RS, Board TN, Gambhir AK, Porter ML and Kay PR: The microbiology of the infected knee arthroplasty. Int Orthop 34: 505-510, 2010

9. Rafiq I, Gambhir AK, Wroblewski BM and Kay PR: The microbiology of infected hip arthroplasty. Int Orthop 30: $532-535,2006$.

10. Theis JC, Gambhir S and White J: Factors affecting implant retention in infected joint replacements. ANZ J Surg 77: 877-879, 2007.

11. Sendi P, Banderet F, Graber P and Zimmerli W: Periprosthetic joint infection following Staphylococcus aureus bacteremia. J Infect 63: 17-22, 2011.

12. Fulkerson E, Valle CJ, Wise B, Walsh M, Preston C and Di Cesare PE: Antibiotic susceptibility of bacteria infecting total joint arthroplasty sites. J Bone Joint Surg Am 88: 1231-1237, 2006.
13. Tsai JC, Sheng WH, Lo WY, Jiang CC and Chang SC: Clinical characteristics, microbiology and outcomes of prosthetic joint infection in Taiwan. J Microbiol Immunol Infect 48: 198-204, 2015.

14. Smith EB, Wynne R, Joshi A, Liu H and Good RP: Is it time to include vancomycin for routine perioperative antibiotic prophylaxis in total joint arthroplasty patients? J Arthroplasty 27 (8 Suppl): S55-S60, 2012.

15. Salgado CD, Dash S, Cantey JR and Marculescu CE: Higher risk of failure of methicillin-resistant Staphylococcus aureus prosthetic joint infections. Clin Orthop Relat Res 461: 48-53, 2007.

16. Arciola CR, Campoccia D, Ehrlich GD and Montanaro L: Biofilm-based implant infections in orthopaedics. Adv Exp Med Biol 830: 29-46, 2015.

17. Muilwijk J, Walenkamp GH, Voss A, Wille JC and van den Hof S: Random effect modelling of patient-related risk factors in orthopaedic procedures: Results from the Dutch nosocomial infection surveillance network 'PREZIES'. J Hosp Infect 62: 319-326, 2006.

18. Barberán J: Management of infections of osteoarticular prosthesis. Clin Microbiol Infect 12 (Suppl 3): S93-S101, 2006.

19. Teterycz D, Ferry T, Lew D, Stern R, Assal M, Hoffmeyer P, Bernard L and Uçkay I: Outcome of orthopedic implant infections due to different staphylococci. Int J Infect Dis 14: e913-e918, 2010.

20. Kuiper JW, Vos SJ, Saouti R, Vergroesen DA, Graat HC, Debets-Ossenkopp YJ, Peters EJ and Nolte PA: Prosthetic joint-associated infections treated with DAIR (debridement, antibiotics, irrigation, and retention): Analysis of risk factors and local antibiotic carriers in 91 patients. Acta Orthop 84: 380-386, 2013.

21. Arciola CR, An YH, Campoccia D, Donati ME and Montanaro L: Etiology of implant orthopedic infections: A survey on 1027 clinical isolates. Int J Artif Organs 28: 1091-1100, 2005.

22. Montanaro L, Speziale P, Campoccia D, Ravaioli S, Cangini I, Pietrocola G, Giannini S and Arciola CR: Scenery of Staphylococcus implant infections in orthopedics. Future Microbiol 6: 1329-1349, 2011.

23. Barnes R and Chetter I: Infection in prosthetic material. Surgery (United Kingdom) 30: 667-672, 2012.

24. Holleyman RJ, Baker P, Charlett A, Gould K and Deehan DJ: Microorganisms responsible for periprosthetic knee infections in England and Wales. Knee Surg Sports Traumatol Arthrosc 24: 3080-3087, 2016.

25. Barrett L and Atkins B: The clinical presentation of prosthetic joint infection. J Antimicrob Chemother 69 (Suppl 1): S25-S27, 2014.

26. Zimmerli W, Trampuz A and Ochsner PE: Prosthetic-joint infections. N Engl J Med 351: 1645-1654, 2004.

27. Moran E, Masters S, Berendt AR, McLardy-Smith P, Byren I and Atkins BL: Guiding empirical antibiotic therapy in orthopaedics: The microbiology of prosthetic joint infection managed by debridement, irrigation and prosthesis retention. J Infect 55: 1-7, 2007.

28. Ip D, Yam SK and Chen CK: Implications of the changing pattern of bacterial infections following total joint replacements. J Orthop Surg (Hong Kong) 13: 125-130, 2005.

29. Cordero-Ampuero J, Esteban J, Garcia-Cimbrelo E, Munuera L and Escobar R: Low relapse with oral antibiotics and two-stage exchange for late arthroplasty infections in 40 patients after 2-9 years. Acta Orthop 78: 511-519, 2007.

30. James PJ, Butcher IA, Gardner ER and Hamblen DL: Methicillin-resistant Staphylococcus epidermidis in infection of hip arthroplasties. J Bone Joint Surg Br 76: 725-727, 1994.

31. Ascione T, Pagliano P, Mariconda M, Rotondo R, Balato G, Toro A, Barletta V, Conte M and Esposito S: Factors related to outcome of early and delayed prosthetic joint infections. J Infect 70: 30-36, 2015.

32. Lutro O, Langvatn H, Dale H, Schrama JC, Hallan G, Espehaug B, Sjursen $\mathrm{H}$ and Engesæter LB: Increasing resistance of coagulase-negative Staphylococci in total hip arthroplasty infections: 278 THA-Revisions due to infection reported to the norwegian arthroplasty register from 1993 to 2007. Adv Orthop 2014: 580359, 2014.

33. Matsumoto T, Ishida K, Tsumura N, Nagai K, Muratsu H, Hida Y, Hiranaka T, Kuroda R and Kurosaka M: Treatment of 50 deep infections after total knee arthroplasty. Orthopedics 38: e529-e535, 2015.

34. Lora-Tamayo J, Murillo O, Iribarren JA, Soriano A, Sánchez-Somolinos M, Baraia-Etxaburu JM, Rico A, Palomino J, Rodríguez-Pardo D, Horcajada JP, et al: A large multicenter study of methicillin-susceptible and methicillin-resistant Staphylococcus aureus prosthetic joint infections managed with implant retention. Clin Infect Dis 56: 182-194, 2013. 
35. Haessler S, Mackenzie T and Kirkland KB: Long-term outcomes following infection with meticillin-resistant or meticillin-susceptible Staphylococcus aureus. J Hosp Infect 69: 39-45, 2008.

36. Arduino JM, Kaye KS, Reed SD and Anderson DJ: Staphylococcus aureus infections following knee and hip prosthesis insertion procedures. Ant Res Infect Control 4, 2015.

37. Kuiper JW, Willink RT, Moojen DJ, van den Bekerom MP and Colen S: Treatment of acute periprosthetic infections with prosthesis retention: Review of current concepts. World J Orthop 5: 667-676, 2014

38. Burger RR, Basch T and Hopson CN: Implant salvage in infected total knee arthroplasty. Clin Orthop Relat Res 105-112, 1991.

39. Wilde AH and Ruth JT: Two-stage reimplantation in infected total knee arthroplasty. Clin Orthop Relat Res 23-35, 1988.

40. Holmberg A, Thórhallsdóttir VG, Robertsson O, W-Dahl A and Stefánsdóttir A: $75 \%$ success rate after open debridement, exchange of tibial insert, and antibiotics in knee prosthetic joint infections. Acta Orthop 86: 457-462, 2015.

41. Ibrahim MS, Raja S, Khan MA and Haddad FS: A multidisciplinary team approach to two-stage revision for the infected hip replacement: A minimum five-year follow-up study. Bone Joint J 96-B: 1312-1318, 2014.

42. Giulieri SG, Graber P, Ochsner PE and Zimmerli W: Management of infection associated with total hip arthroplasty according to a treatment algorithm. Infection 32: 222-228, 2004.

43. Triantafyllopoulos GK, Poultsides LA, Sakellariou VI, Zhang W, Sculco PK, Ma Y and Sculco TP: Irrigation and debridement for periprosthetic infections of the hip and factors determining outcome. Int Orthop 39: 1203-1209, 2015.

44. Bjerke-Kroll BT, Christ AB, McLawhorn AS, Sculco PK, Jules-Elysée KM and Sculco TP: Periprosthetic joint infections treated with two-stage revision over 14 years: An evolving microbiology profile. J Arthroplasty 29: 877-882, 2014.

45. Davis N, Curry A, Gambhir AK, Panigrahi H, Walker CR, Wilkins EG, Worsley MA and Kay PR: Intraoperative bacterial contamination in operations for joint replacement. J Bone Joint Surg Br 81: 886-889, 1999.

46. Mohanty SS and Kay PR: Infection in total joint replacements Why we screen MRSA when MRSE is the problem? J Bone Joint Surg Br 86: 266-268, 2004.

47. James LA, Ibrahim T and Esler CN: Microbiological culture results for the femoral head. Are they important to the donor? J Bone Joint Surg Br 86: 797-800, 2004

48. Bozic KJ, Kamath AF, Ong K, Lau E, Kurtz S, Chan V, Vail TP, Rubash $\mathrm{H}$ and Berry DJ: Comparative Epidemiology of revision arthroplasty: Failed THA poses greater clinical and economic burdens than failed TKA. Clin Orthop Relat Res 473: 2131-2138, 2015.

49. Iarikov D, Demian H, Rubin D, Alexander J and Nambiar S: Choice and doses of antibacterial agents for cement spacers in treatment of prosthetic joint infections: Review of published studies. Clin Infect Dis 55: 1474-1480, 2012.

50. Betz M, Abrassart S, Vaudaux P, Gjika E, Schindler M, Billières J, Zenelaj B, Suvà D, Peter R and Uçkay I: Increased risk of joint failure in hip prostheses infected with Staphylococcus aureus treated with debridement, antibiotics and implant retention compared to Streptococcus. Int Orthop 39: 397-401, 2015.

51. Alijanipour P, Heller S and Parvizi J: Prevention of periprosthetic joint infection: What are the effective strategies? J Knee Surg 27: 251-258, 2014

52. Gálvez-López R, Peña-Monje A, Antelo-Lorenzo R, Guardia-Olmedo J, Moliz J, Hernández-Quero J and Parra-Ruiz J: Elution kinetics, antimicrobial activity, and mechanical properties of 11 different antibiotic loaded acrylic bone cement. Diagn Microbiol Infect Dis 78: 70-74, 2014.

53. Sakamoto $\mathrm{Y}$, Ochiai H, Ohsugi I, Inoue $\mathrm{Y}$, Yoshimura $\mathrm{Y}$ and Kishi K: Mechanical strength and in vitro antibiotic release profile of antibiotic-loaded calcium phosphate bone cement. J Craniofac Surg 24: 1447-1450, 2013
54. Fernandes A and Dias M: The microbiological profiles of infected prosthetic implants with an emphasis on the organisms which form biofilms. J Clin Diagn Res 7: 219-223, 2013.

55. Nishimura S, Tsurumoto T, Yonekura A, Adachi K and Shindo H: Antimicrobial susceptibility of Staphylococcus aureus and Staphylococcus epidermidis biofilms isolated from infected total hip arthroplasty cases. J Orthop Sci 11: 46-50, 2006.

56. Moroski NM, Woolwine S and Schwarzkopf R: Is preoperative staphylococcal decolonization efficient in total joint arthroplasty. J Arthroplasty 30: 444-446, 2015.

57. Liu C, Chen ZJ, Sun Z, Feng X, Zou M, Cao W, Wang S, Zeng J, Wang Y and Sun M: Molecular characteristics and virulence factors in methicillin-susceptible, resistant, and heterogeneous vancomycin-intermediate Staphylococcus aureus from central-southern China. J Microbiol Immunol Infect 48 : 490-496, 2015.

58. Collins J, Rudkin J, Recker M, Pozzi C, O'Gara JP and Massey RC: Offsetting virulence and antibiotic resistance costs by MRSA. ISME J 4: 577-584, 2010.

59. Siddiqui MM, Lo NN, Ab Rahman S, Chin PL, Chia SL and Yeo SJ: Two-year outcome of early deep MRSA infections after primary total knee arthroplasty: A joint registry review. J Arthroplasty 28: 44-48, 2013.

60. McPherson EJ, Patzakis MJ, Gross JE, Holtom PD, Song M and Dorr LD: Infected total knee arthroplasty. Two-stage reimplantation with a gastrocnemius rotational flap. Clin Orthop Relat Res 73-81, 1997.

61. Westberg M, Grogaard B and Snorrason F: Early prosthetic joint infections treated with debridement and implant retention: 38 primary hip arthroplasties prospectively recorded and followed for median 4 years. Acta Orthop 83: 227-232, 2012.

62. Aggarwal VK, Bakhshi H, Ecker NU, Parvizi J, Gehrke T and Kendoff D: Organism profile in periprosthetic joint infection: Pathogens differ at two arthroplasty infection referral centers in Europe and in the United States. J Knee Surg 27: 399-406, 2014.

63. Lourtet-Hascoëtt J, Bicart-See A, Félicé MP, Giordano G and Bonnet E: Is Xpert MRSA/SA SSTI real-time PCR a reliable tool for fast detection of methicillin-resistant coagulase-negative staphylococci in periprosthetic joint infections? Diagn Microbiol Infect Dis 83: 59-62, 2015.

64. Sankar B, Hopgood P and Bell KM: The role of MRSA screening in joint-replacement surgery. Int Orthop 29: 160-163, 2005.

65. Joshy S, Gogi N, Thomas B, Mahale A and Singh BK Delayed onset of deep infection after total knee arthroplasty: Comparison based on the infecting organism. J Orthop Surg (Hong Kong) 15: 154-158, 2007.

66. Tornero E, Garcia-Ramiro S, Martinez-Pastor JC, Bori G, Bosch J, Morata L, Sala M, Basora M, Mensa J and Soriano A: Prophylaxis with teicoplanin and cefuroxime reduces the rate of prosthetic joint infection after primary arthroplasty. Antimicrob Agents Chemother 59: 831-837, 2015.

67. Taha M, Chai F, Blanchemain N, Neut C, Goube M, Maton M, Martel B and Hildebrand HF: Evaluation of sorption capacity of antibiotics and antibacterial properties of a cyclodextrin-polymer functionalized hydroxyapatite-coated titanium hip prosthesis. Int J Pharm 477: 380-389, 2014.

68. El Haj C, Murillo O, Ribera A, Vivas M, Garcia-Somoza D, Tubau F, Cabellos C, Cabo J and Ariza J: Daptomycin combinations as alternative therapies in experimental foreign-body infection caused by meticillin-susceptible Staphylococcus aureus. Int J Antimicrob Agents 46: 189-195, 2015.

69. Ries MD: Vancomycin-resistant Enterococcus infected total knee arthroplasty. J Arthroplasty 16: 802-805, 2001.

70. Soriano A, García S, Bori G, Almela M, Gallart X, Macule F, Sierra J, Martínez JA, Suso S and Mensa J: Treatment of acute post-surgical infection of joint arthroplasty. Clin Microbiol Infect 12: 930-933, 2006 